Revue d'histoire de l'Amérique française

REVUE D.HISTOIRE DE L'AMÉRIQUE FRANÇAISE

\title{
Ségrégation résidentielle et morphologie urbaine, le cas de Shawinigan, 1925-1947
}

\section{Claude Bellavance et François Guérard}

Volume 46, numéro 4, printemps 1993

URI : https://id.erudit.org/iderudit/305146ar

DOI : https://doi.org/10.7202/305146ar

Aller au sommaire du numéro

Éditeur(s)

Institut d'histoire de l'Amérique française

ISSN

0035-2357 (imprimé)

1492-1383 (numérique)

Découvrir la revue

Citer cet article

Bellavance, C. \& Guérard, F. (1993). Ségrégation résidentielle et morphologie urbaine, le cas de Shawinigan, 1925-1947. Revue d'histoire de l'Amérique française, 46(4), 577-605. https://doi.org/10.7202/305146ar
Résumé de l'article

Découvrir les structures de la différenciation spatiale à partir de l'étude de la ségrégation résidentielle dans une ville industrielle de dimension modeste constitue l'objectif principal de ce texte. Shawinigan, née au tournant du siècle avec l'implantation de la grande industrie de ressources, se prête particulièrement bien à une telle entreprise. Dans une première étape, les auteurs subdivisent l'espace résidentiel de la ville en zones homogènes en considérant le statut socioprofessionnel et ethnique des populations qui y habitent. Ces zones sont ensuite classées selon quatre grands types.

Finalement, les auteurs tentent de mettre en relation ces objets construits avec divers facteurs structurants telles la localisation des établissements industriels, la géomorphologie, les intentions des élites politiques et économiques, etc. 


\title{
SÉGRÉGATION RÉSIDENTIELLE ET MORPHOLOGIE URBAINE, LE CAS DE SHAWINIGAN, 1925-1947
}

\author{
CLAUDE BELLAVANCE \\ FRANÇOIS GUÉRARD \\ Centre d'études québécoises
}

Université du Québec à Trois-Rivières

\section{RÉSUMÉ}

Découvrir les structures de la différenciation spatiale à partir de l'étude de la ségrégation résidentielle dans une ville industrielle de dimension modeste constitue l'objectif principal de ce texte. Shawinigan, née au tournant du siècle avec l'implantation de la grande industrie de ressources, se prête particulièrement bien à une telle entreprise. Dans une première étape, les auteurs subdivisent l'espace résidentiel de la ville en zones homogènes en considérant le statut socioprofessionnel et ethnique des populations qui y habitent. Ces zones sont ensuite classées selon quatre grands types. Finalement, les auteurs tentent de mettre en relation ces objets construits avec divers facteurs structurants telles la localisation des établissements industriels, la géomorphologie, les intentions des élites politiques et économiques, etc.

\section{ABSTRACT}

Examining residential segregation in a Canadian city, this paper deals with the spatial differentiation process in Shawinigan, a small industrial town born with the coming of resource-based industries at the turn of the century. The authors first subdivide urban residential space into homogeneous, ethnic and socioprofessional areas. These areas are then classified into four major categories. This partition of residential space is finally examined with regard to the industrial location of establishments, the geomorphological characteristics of the site, political and economic aspirations of local elites, etc.

1. Cet article a été réalisé dans le cadre d'un projet de recherches subventionné par le CRSHC et le Fonds FCAR. Normand Brouillette et Pierre Lanthier en sont les responsables. Qu'ils reçoivent ici nos remerciements pour leur soutien et leurs suggestions. Messieurs Normand Séguin et Serge Courville ont aussi accepté de commenter une version préliminaire de ce texte. Nous les en remercions chaleureusement. 
Aspect fondamental de la dynamique urbaine, la différenciation spatiale est ce processus polymorphe qui conduit à la spécialisation progressive de l'espace urbain ${ }^{2}$. Il est généralement admis que la fonction résidentielle est l'un des principaux vecteurs de la différenciation spatiale au plan social. Au Québec, depuis la fin des années 1960, des travaux d'envergure ont été réalisés sur le phénomène de la ségrégation résidentielle. La plupart de ces études portaient cependant sur Montréal à une époque très récente et étaient peu sensibles à la dimension historique. D'autres recherches, sur Montréal au XIX siècle cette fois, ont par ailleurs permis de traiter de la ségrégation résidentielle ${ }^{3}$ de même que d'autres questions aussi cruciales que la mobilité géographique, le logement et la mortalité infantile ${ }^{4}$. Cet article, qui s'inscrit dans le cadre d'une enquête plus large sur la production de l'espace urbain dans les villes de petite et moyenne dimension au Québec ${ }^{5}$, aborde le processus de la différenciation spatiale par le biais d'une étude de la ségrégation résidentielle à Shawinigan.

Notre lecture de l'espace urbain shawiniganais passe par la délimitation de zones résidentielles homogènes et l'analyse de leurs principales caractéristiques aux plans social et ethnique. Pour ce faire,

2. Voir Marcel Roncayolo, La ville et ses territoires (Paris, Gallimard, 1990).

3. Sherry Olson, «Occupations and Residential Spaces in Nineteenth-Century Montreal», Historical Methods, 22,3 (été 1989): 81-96; Robert Lewis, «The Segregated City. Class Residential Patterns and the Development of Industrial Districts in Montreal, 1861 and 1901», Journal of Urban History, 17,2 (février 1991): 123-152; M. Bellavance et J.-D. Gronoff, «Les structures de l'espace montréalais à l'époque de la Confédération», Cahiers de géographie du Québec, 24,63 (décembre 1980): 363-383. J.-P. Kesteman a posé le phénomène de la différenciation spatiale dans un article concernant les effets des conjonctures économiques sur le développement des espaces socio-économiques de la ville industrielle. Voir «La condition économique vue sous l'angle de la conjoncture économique: Sherbrooke, 1875 à 1914», Revue d'histoire urbaine, 12,1 (juin 1983): 11-28.

4. Pour Montréal, de nombreux travaux traitent de ces questions reliées au phénomène de la ségrégation résidentielle. On peut lire par exemple: P. Thornton, S. Olson et $\mathrm{Q}$. Thuy Thach, «Dimensions sociales de la mortalité infantile à Montréal au milieu du XIX ${ }^{\mathrm{e}}$ siècle», Annales de démographie historique, (1988): 299-325; J.-C. Robert, «Le quartier au milieu du $\mathrm{XIX}^{\mathrm{e}}$ siècle: séjour ou passage?», Habiter la ville. $X V^{e}-X X^{e}$ siècles (Lyon, Presses universitaires de Lyon, 1984), 127-152.

5. On consultera notamment, N. Brouillette, «Le rôle de la Shawinigan Water and Power Co. dans la structuration de l'espace urbain shawiniganais, 1898-1921", Cahiers de géographie du Québec, 34,92 (septembre 1990): 197-208; P. Lanthier et N. Brouillette, «Shawinigan Falls de 1898 à 1930: l'émergence d'une ville industrielle au sein du monde rural», Revue d'histoire urbaine, 19,1 (juin 1990): 42-55; «De la campagne à la ville: formation de la petite bourgeoisie à Shawinigan de 1898 à 1930», G. Bouchard et J. Goy, Famille, économie et société rurale en contexte d'urbanisation (17e-20e siècles) (Chicoutimi et Paris, Centre interuniversitaire SOREP et École des hautes études en sciences sociales, 1990). Concernant Shawinigan, voir aussi, C. Bellavance, R. Hardy, N. Séguin et P. Lanthier, Shawinigan, genèse d'une croissance industrielle au début du XIXe siècle (Trois-Rivières, 1985). 
nous avons procédé à un relevé systématique de la population active et du cadre bâti de cette ville en 1925 et $1947^{6}$. Nous avons voulu aller au-delà d'une simple présentation des formes de la ségrégation résidentielle. Aussi avons-nous confronté les résultats de notre analyse de la fonction résidentielle avec d'autres aspects de la dynamique urbaine. Cette démarche nous a conduits à formuler un certain nombre d'hypothèses pour une éventuelle interprétation d'ensemble de la structuration de l'espace urbain shawiniganais avant 1950.

\section{I - SHAWINIGAN, VILLE D'INDUSTRIES}

Shawinigan est une ville d'industries. Elle offre un exemple frappant de croissance urbaine portée par la grande vague d'industrialisation du premier tiers du $\mathrm{XX}^{\mathrm{e}}$ siècle. Cette agglomération, qui allait devenir pendant quelques décennies l'un des principaux centres de l'industrie chimique au Canada, est littéralement née avec cette nouvelle génération d'entreprises à haute concentration de capital fixe qui furent lancées en Mauricie et dans les autres régions de ressources du Québec à la faveur de la seconde industrialisation (hydroélectricité, pâtes et papiers, électrométallurgie, électrochimie, etc). Pour cette raison, Shawinigan présente un visage différent d'autres centres urbains plus anciens dotés d'un héritage pré-industriel plus ou moins affirmé. Sise entre les contreforts des Laurentides et la rive droite du Saint-Maurice, à proximité de chutes ayant donné leur nom à la ville, Shawinigan se démarque également par son relief accidenté. La rivière, juste avant qu'elle ne précipite sa course en une descente d'environ 38 mètres, rencontre des îles sur son passsage. En cet endroit, elle trace aussi une grande courbe qui forme une sorte de saillie, appelée Pointe-à-Bernard; c'est là que furent érigées les premières habitations et commerces en 1900. Shawinigan, dont l'incorporation date de 1901, a connu une forte croissance démographique au cours de ses premières décennies d'existence. Sa population atteignait ainsi 4265 habitants en 1911 et, en 1925, pas moins de 11500 . Le taux de croissance allait demeurer assez élevé jusqu'aux années $1950^{7}$ alors que s'amorçait le déclin industriel de la ville.

6. Le choix des ces années témoins nous a été en partie dicté par la disponibilité de l'une des deux sources principales sur lesquelles repose notre recherche. Ce choix nous apparaît néanmoins judicieux en ce qu'il correspond en gros: 1 - à un moment de l'histoire de la ville où celle-ci a atteint une taille significative (de l'ordre de 12000 habitants) et où ses principales infrastructures et institutions sont en place; 2 - à la fin d'une période intense de construction domiciliaire qui accompagna et suivit le deuxième conflit mondial et juste avant que ne s'affirme le phénomène de migration vers les banlieues.

7. La population totale de Shawinigan était d'environ 25000 habitants au lendemain de la Seconde Guerre mondiale et de 32169 habitants en 1961. 


\section{CARTE 1}

SHAWINIGAN EN 1925 ET 1947

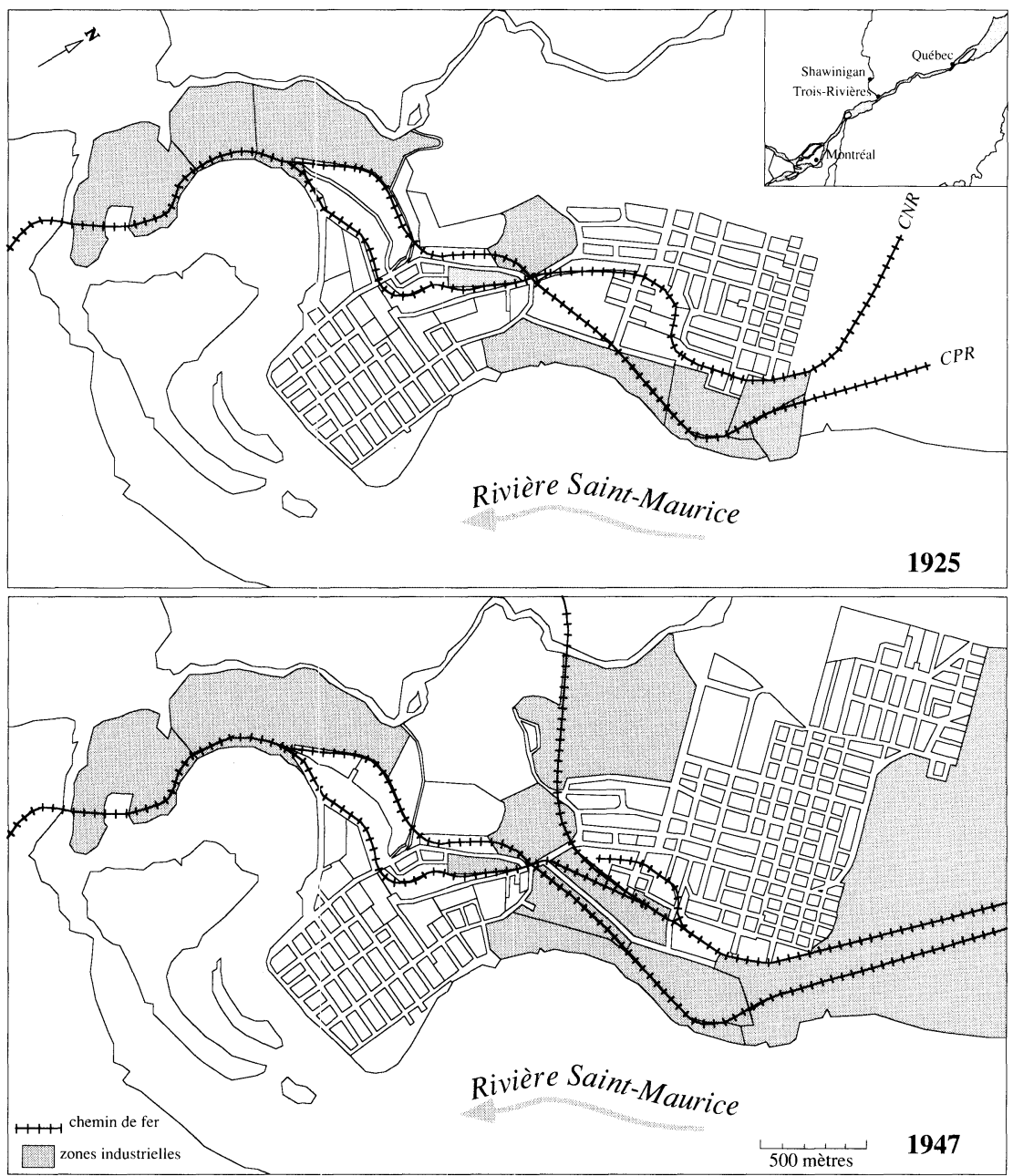

Shawinigan, ville de compagnie? C'est moins certain. Au cours de ses premières décennies d'existence, la ville possédait, il est vrai, plusieurs des traits caractéristiques de ces «company towns» nordaméricaines dans lesquelles l'entreprise-mère fait et défait les conseils municipaux, maintient un contrôle foncier sur une grande partie du territoire et est en mesure d'orienter le développement urbain à sa 
convenance $^{8}$. À bien y regarder cependant, Shawinigan répondrait plutôt mal au modèle. D'abord parce qu'elle n'a jamais été une ville mono-industrielle. Lorsqu'elle entreprit l'aménagement du site des chutes de Shawinigan au tournant du siècle, Shawinigan Water and Power (SWP) n'avait d'autre choix que d'attirer des entreprises industrielles «énergivores» près de ses centrales ${ }^{9}$. L'établissement d'une ville industrielle faisait même partie des projets de ses fondateurs, comme en témoigne la confection d'un plan de développement de la future ville. Dans les années qui suivirent, les activités de promotion industrielle de SWP allaient faire croître encore le nombre d'entreprises clientes établies dans les limites de la ville. Pareille diversification réduisit rapidement l'emprise de la compagnie d'électricité.

Mais si, à l'exception des toutes premières années, Shawinigan n'a pas été sous la tutelle d'une entreprise, elle a par contre été longtemps dominée par sa communauté anglophone, qui, bien que minoritaire ${ }^{10}$, détenait la presque totalité des postes de cadres dans les grandes entreprises. À ces derniers, peu nombreux mais très influents, venait s'ajouter la majorité du personnel de formation scientifique (techniciens, ingénieurs, chimistes, etc.) et une part appréciable du personnel administratif. En ce sens, la société shawiniganaise se conformait parfaitement à la réalité du Québec d'avant la Révolution tranquille, dominé par le capital anglo-canadien ou américain. Bénéficiant de revenus nettement au-dessus de la moyenne, dotée de ses institutions propres, capable d'exercer une influence profonde sur la vie politique locale, cette communauté jouissait d'une forte cohésion, si l'on en prend à témoin la variété et la vigueur des manifestations de sociabilité qui nous sont rapportées par l'hebdomadaire anglophone régional, le St.Maurice Valley Chronicle. Pareille adéquation entre haut statut social et communauté ethnique constitue un avantage majeur, selon nous, pour l'étude de la ségrégation résidentielle. Elle devrait grandement faciliter, en effet, l'analyse de l'une ${ }^{11}$ des dimensions fondamentales de la ségrégation résidentielle: la dimension ethnique.

8. Concernant les villes mono-industrielles du Canada, R. A. Lucas, Minetown, Milltown, Railtown: Life in the Canadian Communities of Single Industry (Toronto, University of Toronto Press, 1971).

9. C. Bellavance, Shawinigan Water and Power. Formation et déclin d'un groupe industriel au Québec, 1898-1963, thèse de doctorat, Université du Québec à Montréal, 1991.

10. On peut estimer à environ $6 \%$ la proportion de la population d'origine anglo-saxonne en 1925, et à 4\% en 1947, d'après les données des recensements du Canada de 1921, 1931, 1941 et 1951 .

11. Nous ne disposons pas de documents nous permettant de traiter avec rigueur de la question du statut familial, une autre dimension importante de la ségrégation résidentielle en milieu urbain. Celle-ci mériterait toutefois qu' on s'y attarde puisque Shawinigan présentait 
L'objectif principal de cet article, nous l'avons dit, est de découvrir, à partir de l'étude de la ségrégation résidentielle, les structures de la différenciation spatiale à Shawinigan en 1925 et 1947 . Une telle démarche s'insère dans l'historiographie aux chapitres des études urbaines et de la géographie historique. Voilà pourquoi il nous apparaît utile, avant de poursuivre avec l'étude du cas de Shawinigan, de jeter un coup d'œil sur les principales méthodes élaborées dans ces champs disciplinaires et de prendre connaissance des problèmes spécifiques que pose l'étude de la ségrégation résidentielle.

\section{2 - FONCTION RÉSIDENTIELLE ET DIFFÉRENCIATION SPATIALE}

Le problème de la ségrégation résidentielle a très tôt occupé une place privilégiée dans les études urbaines, aux États-Unis notamment, et plusieurs modèles ont été proposés pour rendre compte de cet aspect fondamental de la dynamique des villes contemporaines. Depuis les travaux pionniers de E. W. Burgess au cours des années 1920 et de $\mathrm{H}$. Hoyt dans la décennie suivante ${ }^{12}$, la fonction résidentielle s'est affirmée, en effet, comme l'une des clés essentielles pour restituer les cohérences du paysage urbain ${ }^{13}$. La méthodologie s'est affinée depuis et l'on a été en mesure de faire des études de plus en plus ambitieuses sur ce phénomène. Un certain consensus s'est graduellement dégagé (en Amérique du Nord à tout le moins ${ }^{14}$ ) à l'idée que la différenciation de l'espace urbain s'exprime selon trois dimensions principales auxquelles correspondent des formes (ou «patterns») particulières. La première est le statut socio-économique des habitants et partagerait l'espace résidentiel en secteurs (secteur ouest habité par les classes favorisées, secteur est ouvrier, par exemple). La deuxième dimension

\footnotetext{
durant les premières décennies du siècle la répartition par groupes d'âges particulière des villes nouvelles de l'époque, avec une proportion élevée de la population en âge de procréer. Cette population provenait manifestement en majeure partie du milieu rural. Pour en savoir plus sur les origines géographiques des élites shawiniganaises, voir P. Lanthier et N. Brouillette, «De la campagne ....», op. cit.

12. E. W. Burgess a proposé un modèle de développement des villes et de ségrégation résidentielle par zones concentriques, et $\mathrm{H}$. Hoyt un modèle par secteurs radiaux. Voir R. E. Park, E. W. Burgess, R. D. McKenzie, The City (Chicago, University of Chicago Press, 1925); H. Hoyt, The Structure and Growth of Residential Neighbourhoods in American Cities (Washington, 1939).

13. Il n'entre pas dans notre propos de faire un bilan, même sommaire, des principales écoles et des travaux marquants dans le domaine depuis maintenant près de sept décennies. On lira avec intérêt M. Cadwallader, Analytical Urban Geography. Spatial Patterns and Theories (Englewood Cliffs, New Jersey, Prentice Hall, Inc., 1985).

14. M. Roncayolo explique notamment que l'on peut mettre en doute ce modèle dans le cas des villes européennes et asiatiques, op. cit., 120-121.
} 
jugée fondamentale renvoie quant à elle au statut familial, en particulier à la situation des individus et des familles dans leur cycle de vie. On a observé, par exemple, que les familles comportant plusieurs enfants avaient tendance à habiter loin du centre où adultes jeunes et vieux étaient sur-représentés. Vue sous cet angle, la ville apparaît formée d'un certain nombre de zones concentriques, correspondant par ailleurs fréquemment aux grandes phases de son expansion territoriale. Enfin, la troisième dimension, l'ethnicité, laisse entrevoir la formation d'enclaves à l'intérieur du tissu urbain; la forme la plus caractéristique, bien sûr, en est le ghetto. Les villes de l'Amérique du Nord, dont la croissance a été souvent alimentée par des arrivées massives d'immigrants, ont été en partie façonnées suivant les caractéristiques ethniques et raciales de leur population. Les travaux menés au cours des années 1960 et 1970 dans le sillage de l'école de «l'écologie factorielle ${ }^{15}$ » ont confirmé le caractère complémentaire de ces trois modes de lecture du phénomène de la ségrégation résidentielle. Bien plus, au dire de plusieurs, la superposition des «patterns» identifiés ferait apparaître une nouvelle structure, plus complexe, que l'on appelle la mosaïque urbaine.

On a reproché à juste titre à ces diverses approches leur caractère essentiellement descriptif ${ }^{16}$. Au-delà de la diversité des méthodes mises en œuvre, la démarche adoptée dans nombre d'études s'inspirant notamment de l'écologie factorielle se réduit bien souvent en effet à la simple vérification de modèles inspirés des sciences de la nature, sans que les structures de l'espace urbain ne soient véritablement expliquées.

15. L'écologie factorielle vise, par des analyses factorielles variées, à faire ressortir les variables les plus déterminantes dans l'organisation spatiale de la ségrégation résidentielle. Ce type d'études a connu une grande popularité au cours des années 1970 en Europe et en Amérique, notamment au Canada pour des villes comme Toronto, Montréal ou Vancouver. Pour le Québec, voir, Bryn Greer-Wootten, «Le modèle urbain», Ludger Beauregard, dir., Montréal, guide d'excursions (Montréal, Presses de l'Université de Montréal, 1972), 9-31; J.-P. Thouez, «La structure spatiale des caractéristiques socio-économiques de Montréal, 1961», Recherches sociographiques, 14,1 (janvier-avril 1973): 81-116; Peter Foggin et Mario Polèse, La géographie sociale de Montréal en 1971 (Montréal, INRS-Urbanisation, 1976); Louis Guay, «Les dimensions de l'espace social urbain: Montréal 1951, 1961 et 1971», Recherches sociographiques, 19,3 (septembre-décembre 1978): 307-348; Mario Polèse et Serge Carlos, L'écologie factorielle d'un système urbain: une analyse globale des facteurs de différenciation spatiale en milieu urbain pour les principales villes du Canada (Montréal, INRS-Urbanisation, 1978); Louis Guay, «Les dimensions de l'espace social urbain: Québec 1951, 1961 et 1971», Recherches sociographiques, 22,2 (mai-août 1981): 237-255; Céline Le Bourdais et Christine Lefebvre, Spatialisation des composantes ethniques, socio-économiques et familiales à Montréal en 1981 (Montréal, INRS-Urbanisation, 1987).

16. Voir, par exemple, Paul Claval, La logique des villes (Paris, Litec, c1981), 284ss. 
La question du degré de ségrégation est tout aussi fondamentale que celle des formes qu'elle épouse. On s'est demandé, par exemple, si la ville pré-industrielle avait connu des niveaux de ségrégation aussi élevés que ceux que l'on retrouve dans la ville industrielle. Divers travaux semblent indiquer que dans les villes du milieu du XIX ${ }^{\mathrm{e}}$ siècle, la ségrégation résidentielle était peu marquée ${ }^{17}$. Plusieurs chercheurs en ont déduit que c'est l'industrialisation qui a donné l'impulsion nécessaire à la spécialisation accrue de l'espace résidentiel. Quittant un centre-ville de plus en plus malsain en raison de l'activité industrielle et de l'augmentation de la densité de la population, les classes favorisées auraient cherché refuge dans des zones nouvelles à vocation strictement résidentielle, situées en périphérie de la ville. Les élites urbaines auraient veillé à exercer un contrôle sur ces nouveaux quartiers afin d'en conserver les caractères distinctifs. Par la suite, des mouvements de migrations internes analogues auraient gagné tour à tour d'autres groupes sociaux comme certains éléments de la petitebourgeoisie et les cols blancs. Jusqu'au début du siècle cependant, une fraction seulement de la population avait la possibilité matérielle de quitter les quartiers du centre. L'absence de transports en commun efficaces avant les années 1880 , de même que les coûts relativement élevés exigés aux passagers contraignirent les ouvriers, pendant quelques décennies, à demeurer suffisamment près de leur lieu de travail pour s'y rendre aisément à pied ${ }^{18}$.

Mais prendre la mesure du phénomène de la ségrégation résidentielle a posé de sérieux problèmes méthodologiques. Les premiers outils mis en œuvre pour quantifier le phénomène étaient relativement simples, mais ils ne prenaient en compte qu'une petite partie seulement des caractéristiques des populations étudiées. L'emploi de l'informatique a permis de lancer des études beaucoup plus systématiques, faisant intervenir, entre autres, l'analyse factorielle. Toute-

17. Pour ce qui est de l'Amérique du Nord, les travaux influents de Sam Bass Warner notamment indiquent une ségrégation résidentielle peu affirmée: The Private City. Philadelphia in Three Periods of its Growth (Philadelphia, 1968); The Urban Wilderness (New York, 1972). En Angleterre, les résultats des recherches divergent, certains soutenant la thèse d'une ségrégation faible, que d'autres contestent. Voir par exemple David Ward, «Victorian Cities: How Modern?», Journal of Historical Geography, 1 (1975): 135-151; Alan Armstrong, Stability and Change in an English County Town: a Social Study of York, 1805-1851 (Cambridge, 1974); M. P. Conzen, «Historical Geography: Changing Spatial Structure and Social Patterns of Western Cities», Progress in Human Geography, 7 (1983): 88-107.

18. Le logement d'un ouvrier devait semble-t-il se situer dans une zone comprise dans un rayon de deux kilomètres autour du lieu de travail. On pense que vers les années 1910, les coûts du transport en commun avaient cessé de représenter un obstacle à la mobilité des ménages ouvriers. Pour Montréal et Toronto, voir notamment, P.-A. Linteau, «Le transport en commun dans les villes», Normand R. Ball, dir., Bâtir un pays. Histoire des travaux publics au Canada (Montréal, Boréal, 1988), 73-100. 
fois, en dépit des progrès réalisés, les outils d'analyse mis au point ne permettent pas de comparer avec suffisamment de rigueur les niveaux de ségrégation de villes différentes. Les méthodes extensives reposant sur des traitements statistiques sophistiqués se sont révélées, en effet, tout aussi sensibles à la morphologie de chacune des villes que les simples indices de ségrégation utilisés depuis plusieurs décennies déjà $^{19}$. Mais les difficultés ne s'arrêtent pas à ces limites imposées aux études comparatives. Il appert notamment que les diverses méthodes employées pour mesurer le degré de ségrégation résidentielle donnent des résultats très différents selon l'échelle choisie. Un travail reposant, par exemple, sur les données agrégées d'un quartier sera le plus souvent de peu de valeur puisqu'il gommera l'essentiel des différences sociales inscrites dans le tissu urbain. À l'inverse, une étude qui s'en tiendrait uniquement à des unités spatiales très petites risquerait de surévaluer l'importance de ces mêmes phénomènes. En fait, comme nous le verrons dans cet article, l'analyse de la ségrégation résidentielle ne peut être pleinement réussie qu'à partir d'entités spatiales suffisamment vastes et homogènes ${ }^{20}$.

À notre avis, encore trop peu d'études historiques se sont attachées à reconstituer ces cohérences de l'espace urbain à l'échelle appropriée. La subdivision du territoire s'est faite presque toujours en fonction de frontières prétablies (quand on ne s'est pas tout simplement contenté des découpages administratifs). Les géographes apparaissent davantage sensibles à ce type de questions. Sherry Olson, par exemple, a posé le problème dans toute son acuité dans un article récent sur Montréal au XIX ${ }^{\mathrm{c}}$ siècle $^{21}$. Recourant à une méthode associative mise au point par les biologistes pour l'étude du couvert végétal, elle a été en mesure d'établir une typologie des segments de rues de la métropole québécoise en $1861^{22}$ et de reconstituer les structures élémentaires de cette ville au plan de la différenciation sociale $^{23}$. Ce sont d'abord 33 groupes de segments de rues très homo-

19. Ces outils de mesure seront expliqués plus loin.

20. D'autres problèmes surgissent lorsque l'on tente d'appréhender le tissu social urbain par le biais des classes sociales. En effet, bon nombre des intitulés de profession tels qu'ils apparaissent dans la documentation ne permettent pas de préciser la situation des individus dans les rapports de production. Or, on sait l'importance de ces questions pour la compréhension du passage de la ville pré-industrielle à la ville industrielle. C'est l'approche qu'a retenue Robert Lewis dans «The segregated city...», op. cit.

21. Sherry Olson, «Occupations and Residential...», op. cit.

22. Le segment de rue défini par Sherry Olson est formé des deux côtés d'une rue, est délimité par les principales intersections et comprend au moins trente ménages.

23. La même démarche a été utilisée dans un deuxième temps pour tenter un classement des professions déclarées des habitants de la ville non pas en fonction de regroupements préétablis, mais selon le vieil adage «qui s'assemble se ressemble». 
gènes mais offrant peu de continuité sur le plan spatial qui ont été identifiés par l'algorithme de classification. À une échelle intermédiaire, géographiquement beaucoup plus cohérente, $\mathrm{S}$. Olson a découvert 12 types de segments de rues qu'elle a caractérisés en fonction des professions de leurs habitants, puis en prenant en compte d'autres variables telles que la langue, la religion, la complémentarité des métiers en cause, la localisation des établissements industriels, etc. Un dernier niveau de regroupement a mis enfin en évidence une répartition des segments de rues en trois grands groupes correspondant à un secteur aisé très cohésif, au centre-ville, et à une grande zone ouvrière. On le voit, l'intérêt d'une démarche comme celle que propose $\mathrm{S}$. Olson réside notamment dans la mise au point de méthodes permettant d'analyser la ségrégation résidentielle à différentes échelles.

Jusqu'à présent, les historiens au Canada ont pour leur part surtout appréhendé la ségrégation résidentielle dans le cadre de villes préindustrielles ou en voie d'industrialisation ${ }^{24}$, laissant la ville du $\mathrm{XX}^{\mathrm{e}}$ siècle aux urbanistes, sociologues ou géographes. Les villes industrielles de petite et moyenne dimensions, en particulier, sont très mal connues sous ce rapport. Or, elles ne manquent pas d'intérêt. Si l'on excepte les micro-études portant sur un quartier, une paroisse ou une section du territoire urbain, les recherches sur la différenciation spatiale dans les grandes villes passent presque obligatoirement par des méthodes d'échantillonnage plus ou moins élaborées ${ }^{25}$ ou sur l'usage de données déjà agrégées, ce qui comporte des inconvénients majeurs: risques d'erreurs en ce qui a trait à la représentativité des secteurs choisis, difficulté de bien saisir le rôle des nombreuses institutions et entreprises, etc. Mais, on l'aura deviné, nous pensons surtout ici aux effets négatifs d'un découpage non homogène du territoire. En choisissant une ville de dimension modeste, il devient possible, au contraire, de considérer l'ensemble du territoire et de la population qui s'y trouve et, pour autant que l'on adopte une démarche appropriée, d'établir une subdivision du territoire qui rende compte des clivages et de la trame du tissu urbain.

24. Voir J.-P. Kesteman, op. cit.; John Hare, «La population de la ville de Québec, 17951805», Histoire sociale, 7,13 (mai 1974): 23-47; M. Bellavance et J.-D. Gronoff, «Les structures de l'espace montréalais...", op. cit.; Ian Davey et Michael Doucet, "The Social Geography of a Commercial City, ca.1853:, M. Katz, The People of Hamilton, Canada West: Family and Class in a Mid-Nineteenth Century City (Cambridge, Harvard University Press, 1982), 319-342.

25. Voir par exemple l'ouvrage d'Olivier Zunz, Naissance de l'Amérique industrielle. Détroit, 1880-1920 (Paris, Aubier, «collection historique», 1983). L'auteur retient plus de cent vingt unités d'analyse composées chacune de 6 sections de rues, 4 de celles-ci formant un îlot avec en plus deux façades de rues parmi les îlots adjacents. 


\section{3 - DÉCOUVRIR LES STRUCTURES DE BASE DE L'ESPACE RÉSIDENTIEL SHAWINIGANAIS}

Les sources

Cette étude s'appuie principalement sur le dépouillement intégral de deux sources complémentaires ${ }^{26}$ : les plans d'assurances préparés en 1925 et 1947 par Underwriter's Survey Bureau Ltd et les bottins d'adresses publiés à Shawinigan les mêmes années ${ }^{27}$. Destinés à l'origine aux assureurs désireux d'établir le niveau des primes de leurs clients en fonction des risques encourus, les plans d'assurances sont d'une très grande richesse et permettent une reconstitution précise du bâti au moment de leur réalisation ${ }^{28}$. Y sont inscrits: l'emplacement, le périmètre et l'adresse de tous les bâtiments érigés à l'intérieur des limites de la ville, le ou les usages auxquels ils sont destinés, le nombre d'étages, les matériaux de construction utilisés tant pour la charpente que pour le recouvrement, ainsi qu'une foule d'autres indications (coupe-feu, tracé des rues et des parcs, lampadaires, topographie, etc.) Quant aux bottins d'adresses, ils nous permettent de reconstituer l'essentiel de la population active de la ville. Outre le nom et l'adresse des individus, ils fournissent le métier ou la profession. Cette recension n'est pas exhaustive, puisqu'elle exclut les personnes n'exerçant pas un métier ou une profession (ménagères, enfants d'âge scolaire, etc.). L'unité de base du dépouillement des plans d'assurances a été le bâtiment. Le jumelage des informations provenant des bottins avec celles des plans d'assurances a été réalisé grâce aux numéros civiques. Toutefois, il a été jugé plus commode de n'associer les individus au cadre bâti qu'au niveau des segments d'îlot (chaque segment d'îlot représente ici une façade de rue d'un îlot). Ce travail a été réalisé pour l'essentiel de façon automatique à l'aide des outils de programmation d'un logiciel de base de données relationnelle. Au total, $11254(95,4 \%)$ des 11796 individus apparaissant aux bottins d'adresses ${ }^{29}$ ont pu être ainsi associés à l'un ou l'autre des 645 segments d'îlot habités pour les deux années témoins.

26. Nous remercions France Normand et Andrée Thibeault qui ont effectué la saisie sur ordinateur d'une partie des données utilisées dans cet article.

27. Signalons que les plans d'assurances et les bottins viennent en partie combler l'absence, à Shawinigan, de rôles d'évaluation ou de perception, malheureusement détruits.

28. On trouvera une description du contenu des plans d'assurances dans R. J. Hayward, «Insurance Plans and Land Use Atlases: Sources for Urban Historical Research», Revue d'histoire urbaine, 1 (mai 1973): 2-9.

29. Nous croyons toutefois qu'une partie non négligeable de la population active, peutêtre 15\%, nous échappe en 1925. Ces individus que l'on a omis d'inscrire dans les bottins d'adresses semblent surtout appartenir au groupe des cols bleus non qualifiés et habiter dans un secteur particulier où ces derniers sont par ailleurs nettement sur-représentés. 
TABLEAU 1

SHAWINIGAN, INDICES DE DISSIMILITUDE POUR LES PRINCIPALES CATÉGORIES SOCIO-PROFESSIONNELLES, POPULATION ACTIVE, 1925 ET 1947 (UNITÉ DE BASE: LE SEGMENT D'ÎLOT)*

\begin{tabular}{|c|c|c|c|c|c|c|}
\hline 1925 & cols bleus & cols blancs & $\begin{array}{l}\text { professions } \\
\text { libérales et } \\
\text { scientifiques }\end{array}$ & $\begin{array}{l}\text { marchands } \\
\text { et artisans }\end{array}$ & $\begin{array}{l}\text { personnel } \\
\text { dirigeant }\end{array}$ & $(n)$ \\
\hline cols bleus & 0,0 & & & & & $(1616)$ \\
\hline cols blancs & 63,5 & 0,0 & & & & $(75)$ \\
\hline professions libérales et scientifiques & 64,6 & 48,1 & 0,0 & & & $(85)$ \\
\hline marchands et artisans & 40,9 & 56,1 & 59,7 & 0,0 & & $(184)$ \\
\hline personnel dirigeant & 39,2 & 50,7 & 53,6 & 53,1 & 0,0 & $(228)$ \\
\hline \multicolumn{7}{|l|}{1947} \\
\hline cols bleus & 0,0 & & & & & (4 313) \\
\hline cols blancs & 55,9 & 0,0 & & & & $(376)$ \\
\hline professions libérales et scientifiques & 64,3 & 56,3 & 0,0 & & & $(308)$ \\
\hline marchands et artisans & 43,7 & 54,0 & 56,4 & 0,0 & & $(423)$ \\
\hline personnel dirigeant & 52,9 & 45,4 & 49,5 & 52,6 & 0,0 & $(405)$ \\
\hline
\end{tabular}

Le phénomène de la ségrégation résidentielle: une première mesure globale

En études urbaines, on a eu fréquemment recours à deux instruments relativement simples pour mesurer le niveau de ségrégation résidentielle: les indices «de ségrégation» et «de dissimilitude» («dissimilarity»). Le premier livre une mesure globale de la propension des habitants d'un groupe quelconque à habiter les mêmes lieux. Le second met en relation chacun des groupes avec non plus le reste de la population, mais avec chacun des autres groupes. Il vise non seulement à établir quels groupes sociaux ou ethniques sont les plus ségrégés, mais aussi à révéler les degrés d'affinité des groupes entre eux.

Nous avons voulu vérifier ce que ces indices pouvaient révéler sur le phénomène de la ségrégation résidentielle à Shawinigan. Pour le calcul des indices de dissimilitude, nous avons classé les 540 intitulés de profession recensés dans les bottins d'adresses en grandes catégories socioprofessionnelles. Il appert que le phénomène de la ségrégation résidentielle ne se laisse pas facilement saisir avec un tel outil. Certes, des tendances très nettes à la ségrégation ressortent. Ainsi, au tableau 1, un indice de dissimilitude particulièrement élevé sépare les travailleurs cols bleus du groupe des professions libérales et du personnel de formation scientifique, et en 1925 un indice presque équivalent caractérise les relations entre cols bleus et cols blancs. Par 
contre, on s'étonnera qu'en 1925, ce sont les membres des catégories «cols bleus» et «personnel dirigeant» qui semblent présenter la plus grande propension à vivre dans une même entité spatiale (un segment d'îlot). Dans l'ensemble, tous les groupes paraissent assez fortement ségrégés entre eux. En fait, la principale difficulté réside ici dans le choix de l'unité spatiale de base. Cette méthode présente en effet le défaut d'être très sensible aux phénomènes d'échelle. Selon que les calculs sont faits à partir d'une petite entité comme le segment d'îlot ${ }^{30}$ ou d'une zone plus vaste (une unité de recensement, par exemple), les indices obtenus s'avéreront plus ou moins grands. Il paraît par conséquent difficile de comparer ces résultats avec ceux d'autres études $^{31}$. Enfin, si de tels indices révèlent des tendances qui s'expriment à l'échelle de la ville, ils gomment tout le problème des rapports des groupes sociaux à l'espace.

\section{Définir des zones homogènes}

Le simple calcul des indices de dissimilitude pour le cas de Shawinigan s'avérait donc insatisfaisant. En fait, il nous est très tôt apparu qu'il fallait aborder le problème de la ségrégation résidentielle par une autre voie qui tiendrait compte cette fois des continuités inscrites dans le paysage urbain et mènerait à la définition d'aires résidentielles plus vastes et homogènes.

Ne pouvant faire nôtre une quelconque division préétablie du territoire shawiniganais ${ }^{32}$ pas plus que nous ne pouvions faire porter l'analyse au niveau des entités de base ayant servi au dépouillement, nous avons dû créer de toutes pièces des zones que nous avons voulues les plus homogènes possible. Quelques précisions s'imposent en

30. C'est ici le cas. Or, il arrive fréquemment que l'on dénombre moins de dix individus dans un segment d'îlot.

31. Ce problème a été signalé par différents auteurs, dont David Ley, A Social Geography of the City (Cambridge, Harper \& Roe, 1983), 60.

32. Comme nous l'avons dit plus haut, bon nombre d'études portant sur la ségrégation résidentielle au $\mathrm{XX}^{\mathrm{e}}$ siècle adoptent un découpage de l'espace urbain calqué sur des divisions administratives du territoire. Ce choix s'explique le plus souvent par le fait que l'analyse repose sur l'exploitation de données déjà regroupées par unités de recensement. L'usage des données de recensements nationaux permet au chercheur de faire intervenir de nombreuses variables qu'il serait bien en peine de réunir autrement pour l'ensemble d'une ville. L'impossibilité d'accéder aux données nominatives avant un certain délai oblige toutefois à adopter, pour les études touchant au $\mathrm{XX}^{\mathrm{e}}$ siècle, les divisions territoriales selon lesquelles les données ont été colligées. Or, rien ne garantit qu'une pareille division corresponde aux véritables cohérences du paysage urbain (que ce soit au plan architectural, social, ethnique ou autre). S'agissant d'une enquête portant sur des grandes villes divisées en centaines d'unités de recensement, les biais induits seront plus ou moins importants selon les objectifs visés. Dans le cas d'une ville de taille plus modeste, cette procédure s'avère tout simplement impraticable. 
ce qui a trait à cette notion d'homogénéité et aux principes qui nous ont guidés pour établir une partition de l'espace résidentiel shawiniganais.

La première étape a consisté à définir un cadre de classement pour les 540 intitulés de professions recensés aux bottins. Il a fallu ensuite déterminer lesquelles des 18 catégories socioprofessionnelles ${ }^{33}$ retenues constituaient les principaux vecteurs de la différenciation spatiale. Pour y parvenir, nous avons eu recours à une méthode statistique appelée «calcul des résidus standardisés». Il s'agit, pour chacune des entités spatiales de base (segment d'îlot) de la ville et pour chaque catégorie socioprofessionnelle, de mesurer l'écart entre la distribution effective de la population et une distribution théorique sans aucune ségrégation, c'est-à-dire qui respecterait, en chacune des entités spatiales de base, la répartition générale de la population en groupes socioprofessionnels. Les valeurs obtenues ont été ensuite transformées afin de pouvoir les comparer ${ }^{34}$. Ce test a permis d'identifier quatre catégories socioprofessionnelles dont la répartition spatiale paraissait particulièrement significative. Ce sont: 1 - les cols bleus non qualifiés; 2 - les cols blancs; 3 - le personnel de formation scientifique (techniciens, ingénieurs et chimistes); et 4 - les cadres supérieurs.

Disposant de ces outils d'analyse, nous étions alors en mesure de commencer le travail proprement dit de délimitation des zones résidentielles de la ville en 1925 et en 1947. Pour ce faire, nous avons d'abord établi un seuil ${ }^{35}$ au-delà duquel une catégorie socioprofessionnelle serait considérée sur-représentée dans un segment d'îlot donné. Chacun des segments d'îlot a, par la suite, été classé en fonction de la sur-représentation des quatre catégories socioprofessionnelles

33. En voici la liste: travailleurs cols bleus non qualifiés, cols bleus qualifiés ou semiqualifiés, cols bleus aux qualifications indéterminées, cols blancs, travailleurs autres ou indéterminés, membres des professions libérales traditionnelles, personnel de formation scientifique (ingénieurs surtout), professionnels autres, personnel dirigeant supérieur ou intermédiaire, personnel dirigeant de statut inférieur, propriétaires de PME, personnel dirigeant de statut indéterminé, marchands et commerçants considérés comme propriétaires de leurs commerces, artisans, marchands et commerçants de statut indéterminé, clergé, catégories «autres» et «indéterminés». Le classement des catégories socioprofessionnelles est un travail complexe. Diverses méthodes ont été proposées par les historiens. Pensons, par exemple, à G. Bouchard et C. Pouyez, «Les catégories socioprofessionnelles: une nouvelle grille de classement», Labour/Le travail, 15 (printemps 1985): 145-163. Ces grilles n'étaient cependant pas applicables à notre corpus.

34. Elles ont été standardisées, de sorte que la moyenne de chacune des séries obtenues (ici la différence entre distribution théorique et distribution réelle en catégories socioprofessionnelles selon le segment d'îlot) soit de zéro et l'écart-type égal à un. Nous avons non seulement analysé ces données transformées en tableau, mais les avons aussi reportées sur des cartes choroplèthes.

35. Ce seuil a été fixé̉ empiriquement à 0,4 écart-type. 
CARTE 2

SHAWINIGAN: LES ZONES RÉSIDENTIELLES EN 1925 ET 1947

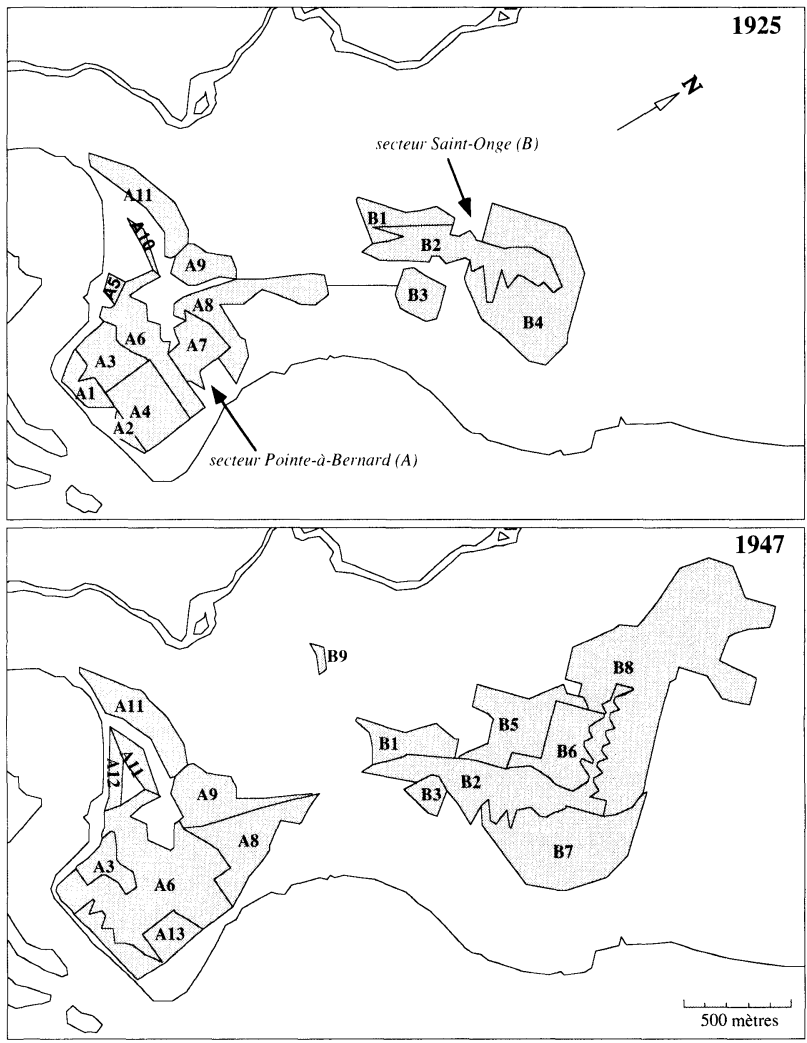

choisies. Ainsi, certaines de ces unités spatiales apparurent-elles privilégiées par les cadres supérieurs, d'autres par les cols bleus non qualifiés, d'autres à la fois par les cols blancs et le personnel de formation scientifique, etc. Ces traits spécifiques ont ensuite été reportés sur deux cartes choroplèthes (1925 et 1947) sur lesquelles il devenait possible d'identifier un certain nombre d'espaces adjacents partageant essentiellement les mêmes caractéristiques ${ }^{36}$.

36. Pour établir les limites de chacune des zones, nous avons procédé par annexions successives d'unités spatiales de base (segments d'îlots), en partant des regroupements les plus manifestement homogènes et en étendant les zones jusqu'à la rencontre d'unités ne correspondant pas aux caractéristiques de la zone ainsi formée. Dans certains cas, d'autres éléments que les seules caractéristiques socioprofessionnelles ont aidé à fixer les frontières entre zones: continuités ou discontinuités liées à des traits architecturaux, à la présence d'axes ferroviaires ou routiers, à une topographie accidentée. 
Au total 31 zones, de tailles fort variables, ont été ainsi définies, soit 15 pour 1925 et 16 pour 1947 (carte 2). Ces zones appartiennent à l'un ou l'autre des deux grands secteurs de la ville: le secteur de la Pointe-à-Bernard (les entités commençant par la lettre A) et le secteur Saint-Onge (les entités commençant par la lettre B). Certaines, comme les zones A5 et B9, ne renferment que quelques îlots seulement alors que d'autres, les zones B7 et B8 par exemple, occupent une vaste portion du territoire. La partition de l'espace urbain shawiniganais qui nous est donnée ici laisse entrevoir des permanences (la zone A11, par exemple, dont le tracé reste à peu près inchangé entre 1925 et 1947) mais aussi des transformations majeures (zone A6 en particulier) qu'il nous faudra tenter de mieux comprendre.

\section{4 - LA STRUCTURATION DE L'ESPACE URBAIN À SHA WINIGAN ENTRE 1925 ET 1947}

Le découpage en zones du territoire shawiniganais pour les années 1925 et 1947 nous permet maintenant d'en faire une lecture d'ensemble en fonction des caractéristiques socioprofessionnelles et linguistiques de la population, et de fournir des éléments pour une synthèse de l'évolution de la ville au cours de cette période.

\section{Une typologie des zones résidentielles}

La meilleure façon de saisir les traits essentiels de l'espace résidentiel consiste à établir une typologie des zones que nous venons de tracer. Il s'agit, en bref, de classer chacune des zones de la ville en fonction de caractéristiques communes. Pour y parvenir, nous avons choisi de faire appel à une méthode dite de «classification ascendante hiérarchique ${ }^{37}$ ». Les quatre catégories socioprofessionnelles ayant servi à la partition de l'espace de même que le groupe linguistique d'appartenance ${ }^{38}$ ont été pris en compte par l'algorithme de classi-

37. La classification ascendante hiérarchique est une méthode de classification couramment utilisée en statistiques. Le tableau des valeurs obtenues pour chacune des 5 variables retenues ici (travailleurs cols bleus non qualifiés, cols blancs, personnel de formation scientifique, cadres supérieur ou intermédiaire et membres des professions libérales) peut être représenté mathématiquement par un nuage de points. La classification ascendante hiérarchique consiste, pour l'essentiel, à calculer la distance euclidienne entre ces points dans un espace à «n» dimensions (pour «n» variables) afin de classer et de regrouper les individus (au sens statistique) partageant essentiellement les mêmes caractères. Pour plus de détails voir, Groupe Chadule, Initiation aux pratiques statistiques en géographie (Paris, Masson 1987), 150ss, et Paul F. Velleman, Data Desk Statistics Guide (Ithaca, Data Description Inc., 1988), 2: chapitre 24.

38. Ne disposant pas d'informations plus précises, le groupe linguistique (anglophone, francophone, autres et indéterminé) a été déterminé à partir de la consonance des noms d'individus. Cette méthode, il va sans dire, n'est pas sans comporter certains risques d'erreurs. Nous croyons toutefois qu'il s'agit d'une approximation valable. Les deux séries d'histogrammes en appendice présentent les sur/sous-représentations de principales catégories socioprofessionnelles et groupes linguistiques par zones et par types de zones. 
FIGURE 1

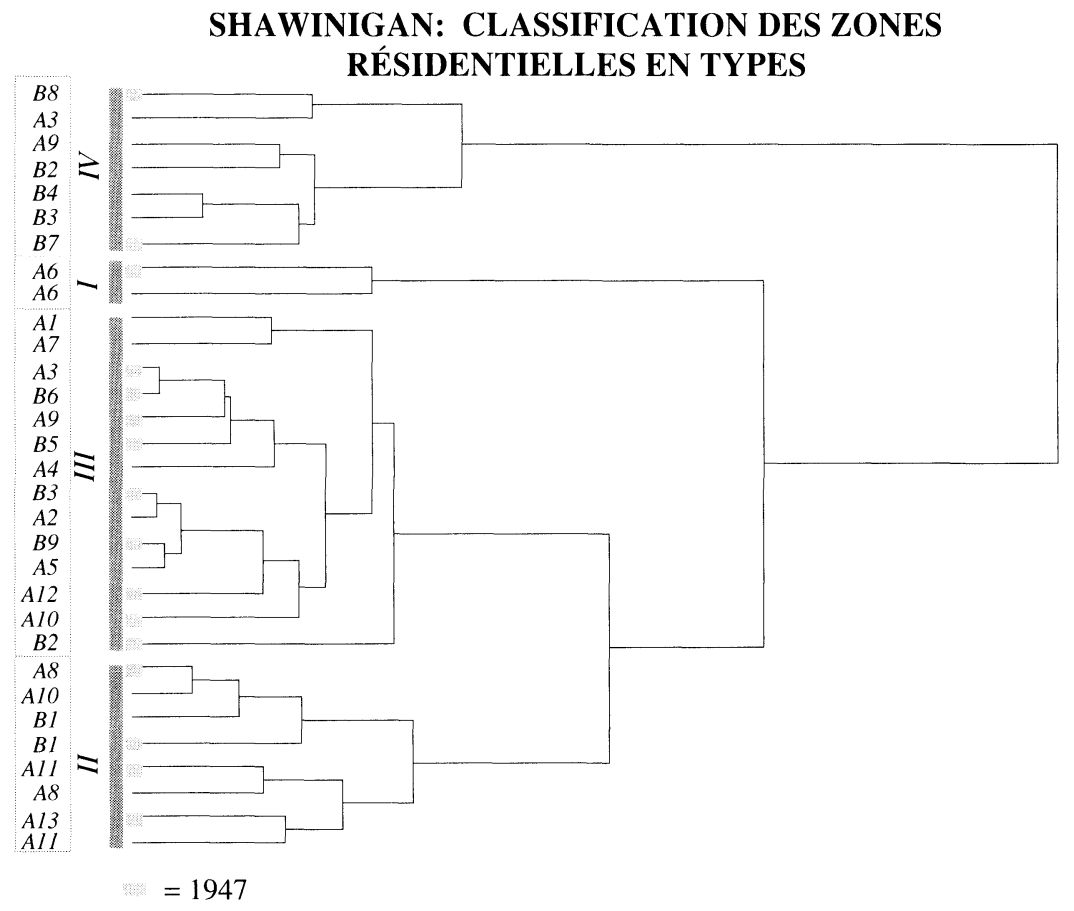

fication (figure 1). Les 31 zones résidentielles que comporte Shawinigan en 1925 et 1947 ont pu être ainsi classées en 4 grands types (I, II, III et IV). La zone centrale de la Pointe-à-Bernard (A6) représente un type à elle seule (le type I). Il s'agit, tant en 1925 qu'en 1947, du centre-ville de Shawinigan. Cette zone commerciale connaît les plus hauts taux de ségrégation négative envers les travailleurs cols bleus non qualifiés, et est le lieu de résidence privilégié des travailleurs cols blancs ainsi que des membres des professions libérales. Les zones de type II, au nombre de 4 pour chacune des deux années, sont caractérisées quant à elles par une forte représentation d'anglophones, de cadres supérieurs et de personnel de formation scientifique. Il est intéressant de constater que ces zones, les plus aisées de la ville, se démarquent aussi du point de vue architectural: habitations spacieuses souvent de style européen, etc. Les zones de type III, les plus nombreuses ( 5 en 1925 et 9 en 1947), ne se laissent pas aisément caractériser. La plupart des catégories socioprofessionnelles identifiées comme responsables des clivages sur le plan spatial ne présentent sur les histogrammes (appendices 1 et 2) qu'assez peu de relief. 
Peut-on aller jusqu'à qualifier ce type de zone de secteurs neutres? L'idée paraît acceptable de prime abord, surtout lorsque l'on considère les résultats plus nettement tranchés obtenus avec les autres types de zones. Un examen plus attentif révèle tout de même certaines constantes. On remarque, en effet, une tendance à la sur-représentation (légère ou moyenne) des francophones de même que des travailleurs cols bleus qualifiés, alors que les catégories «personnel de formation scientifique», «cadres supérieurs» et «professions libérales» sont, au contraire, sous-représentées (faiblement ou moyennement). Si l'on peut globalement associer ce type de zone aux couches les plus qualifiées des ouvriers d'usines, il faudra quand même garder à l'esprit que le phénomène de la ségrégation y est généralement peu affirmé, et que les zones de cette catégorie sont souvent nettement moins homogènes que les autres ${ }^{39}$. Le quatrième type de zone (IV) est d'abord et avant tout celui des cols bleus non qualifiés qui y résident nombreux ${ }^{40}$. La population francophone y est aussi presque toujours nettement sur-représentée (zones B7, B8, etc.). Ces zones, qui occupent de larges portions du territoire shawiniganais, donnent l'impression d'une grande homogénéité.

Le report sur la carte 3 de cette typologie des zones est à son tour extrêmement intéressant. L'opposition entre les deux parties de la ville y apparaît clairement, surtout en 1947, alors que le grand secteur de la Pointe-à-Bernard (les entités commençant par la lettre A) voit s'étendre la zone du centre-ville (A6) et changer de nature les deux anciennes zones de type IV: à forte prédominance de cols bleus non qualifiés francophones en 1925, les zones A3 et A9 sont passées à la catégorie III une vingtaine d'années plus tard. Mais les transformations sont peut-être encore plus nombreuses dans l'autre grand secteur de la ville, le secteur Saint-Onge (les entités commençant par la lettre B), qui connaît une expansion par ailleurs considérable. C'est là que se concentre l'essentiel de la population ouvrière de Shawinigan en 1947. Si, en 1925, les couches les moins favorisées de la population de la ville étaient sur-représentées dans quatre des cinq zones de ce grand secteur, il en va tout autrement en 1947. Certes, elles teignent de noir une portion importante de la carte choroplèthe. Mais d'autres catégories de citadins sont venues s'y installer aussi, comme en

39. L'une de ces zones de type III, B2 en 1947, se distingue quelque peu, aussi bien dans les histogrammes présentés en appendice que dans la classification hiérarchique ascendante. Seules les professions libérales y sont sur-représentées. Cette zone constitue en fait un deuxième secteur commercial en voie de formation, en transition pourrait-on dire, caractérisé par l'introduction de services privés dans une zone antérieurement habitée surtout par des cols bleus.

40. En 1947 les travailleurs cols bleus qualifiés ou semi-qualifiés sont aussi surreprésentés dans ce type de zone. 
CARTE 3

LES ZONES RÉSIDENTIELLES À SHAWINIGAN: TYPOLOGIE (1925 ET 1947)

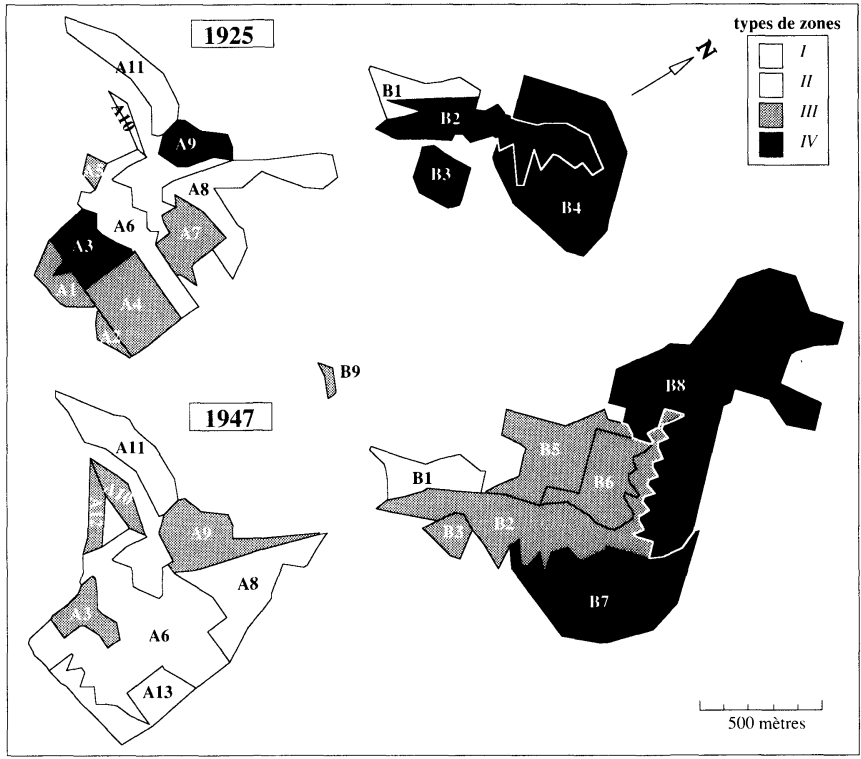

témoigne cette percée de quatre zones de type III. Mais il y a plus. Tout se passe, en effet, comme si les couches les moins favorisées de la population avaient été refoulées vers les marges de l'espace urbain shawiniganais. Les nouvelles zones B7 et B8, constituées pour une bonne part il est vrai à partir de l'ancienne zone B4, comportent nombre d'habitations de qualité médiocre. De plus, une part non négligeable des lotissements qui les composent sont encore à peu près vacants ${ }^{41}$.

La comparaison des zones résidentielles révèle aussi de lourdes permanences, comme ces espaces privilégiés, apparemment réservés aux élites économiques, que sont les zones de type II. Ici, peu de changements en vingt-deux ans sinon que l'on a troqué la petite zone A10 contre les beaux terrains situés au bord du Saint-Maurice et compris dans la zone A13. Cette dernière se démarque quelque peu des autres zones de type II en ce qu'elle est plus souvent habitée par des membres des professions libérales que par le personnel

41. Cela est particulièrement fréquent dans la partie la plus au nord de la zone B8 où il arrive qu'un îlot ne recèle qu'une ou deux habitations. 
scientifique et technique des entreprises. Si elle connaît le deuxième plus haut taux de ségrégation positive pour les cadres supérieurs et intermédiaires, elle apparait par contre comme la moins anglophone des zones de sa catégorie, et est la seule à accueillir une proportion importante de cols blancs. Les zones A11 (la rue Maple et ses demeures cossues) et $\mathrm{A} 8$ (où l'on rencontre la majeure partie des équipements culturels de la communauté anglophone) représentent certainement les noyaux durs de la ségrégation sociale et ethnique à Shawinigan. La zone B1, seule de sa catégorie dans la partie nord de la ville, apparaît quant à elle presque étrangère à son environnement immédiat. Une topographie qui la vouait à un relatif isolement ${ }^{42}$, mais surtout la construction par SWP en 1916, d'habitations modèles destinées à loger du personnel cadre et des techniciens ont fait de cette section du secteur Saint-Onge une sorte de prolongement des «beaux quartiers» de la Pointe-à-Bernard.

\section{Quelques propositions pour une interprétation d'ensemble}

Considéré du point de vue de sa morphologie la plus élémentaire, l'espace résidentiel shawiniganais nous apparaît donc nettement différencié. Les groupes sociaux et ethniques se répartissent spatialement selon une logique qui reste toutefois encore largement à découvrir. Nous n'avons pas l'intention de rendre compte ici de manière définitive, ni même approfondie, du processus global de la production de l'espace shawiniganais. Trop d'éléments manquent encore, dont une connaissance fine des éléments architecturaux du cadre bâti, des informations précises sur le lieu de travail, sur le prix des loyers, etc. Il est néanmoins possible, en partant notamment des acquis de notre recherche, de présenter, avant de conclure, un certain nombre de propositions pour une interprétation d'ensemble.

La fonction résidentielle n'est qu'une des grandes fonctions urbaines. Dans une certaine mesure, on pourrait soutenir qu'elle se trouve souvent subordonnée à d'autres dimensions de la vie des villes, tels les activités industrielles et commerciales, ou les services municipaux. Les configurations physique et sociale des diverses zones résidentielles sont directement influencées, en effet, par le tracé des infrastructures de transport et de communication, la localisation des entreprises, la qualité et la disponibilité des services publics et privés, etc. La topographie impose ses contraintes elle aussi mais peut tout aussi bien être mise à profit par certains groupes sociaux désireux de s'approprier des espaces résidentiels fortement différenciés. Les inten-

42. Le relief est en effet très marqué sur les trois quarts du pourtour de cette zone. 
tions des promoteurs enfin - surtout dans le cas d'une ville nouvelle, largement planifiée, comme Shawinigan - doivent être prises en considération. Tous ces éléments structurants interagissent ou se superposent en fonction des impératifs du moment ainsi que des intérêts et des valeurs des élites économiques et politiques de la ville.

Les structures de base de l'espace social shawiniganais ont d'abord été façonnées par la localisation des établissements industriels et par le tracé de la voie ferrée du Shawinigan Falls Terminal Railway, une filiale de SWP qui reliait les grandes entreprises manufacturières de la ville aux réseaux nationaux de chemins de fer (se reporter à la carte 1). Rail et industries ont en effet partagé Shawinigan en deux grandes sections tout à fait distinctes qu'une seule voie de communication reliait directement: le passage entre l'extrémité nord de la rue des Cèdres - l'un des principaux axes de la Pointe-à-Bernard (secteur A) - et la rue Saint-Marc qui traverse le secteur Saint-Onge (B). Les concepteurs du devis initial n'avaient pas prévu cette division fondamentale de la ville, ayant plutôt privilégié un développement de l'habitat en continu le long de la rivière ${ }^{43}$. Mais il semble bien que l'accès au Saint-Maurice ait été jugé prioritaire pour les entreprises de produits chimiques, car c'est tout un secteur résidentiel qui fut sacrifié dès le tout début du siècle pour y loger les usines de carbure de calcium et de ses dérivés. Quant au tracé de chemin de fer qui passe entre les deux grandes parties de la ville, il utilise en fait la seule voie disponible pour rejoindre d'importants établissements industriels sans rencontrer d'obstacles naturels.

Cette nette démarcation entre les deux grandes sections de la ville apparaît comme la structure la plus élémentaire de l'espace urbain à Shawinigan. Elle a favorisé l'émergence d'une nouvelle artère commerçante (la rue Saint-Marc) ainsi que le dédoublement de divers services municipaux (postes de police et de pompiers, parcs, etc.). Les services publics à Shawinigan se sont toutefois développés de manière très inégale dans ces deux grands secteurs. Par exemple, en 1919 encore, les habitants de toute une partie du secteur Saint-Onge (B) ne disposaient pas du service d'aqueduc et d'égout. Rien d'étonnant alors à ce qu'en 1925 (carte 3), toutes les zones de cette partie de la ville à l'exception de la zone modèle B1 appartiennent - selon la typologie adoptée plus haut - à la catégorie IV, catégorie caractérisée, on s'en souviendra, par l'abondance de cols bleus non qualifiés francophones. Il est remarquable enfin de constater que ces zones sont aussi celles dont le développement n'a fait l'objet initialement d'aucune

43. Ce plan a été reproduit dans N. Brouillette, «Le rôle...», op. cit. 
planification. En effet, le plan initial de la compagnie d'électricité couvrait la partie de la Pointe-à-Bernard, et l'essentiel des habitations de la zone B1, seule de son type dans le secteur Saint-Onge, ont été construites par SWP.

À une échelle intermédiaire, celle des zones identifiées dans cet article, l'impact de la localisation des établissements industriels et des infrastructures de transport se fait moins direct. Certes, elles façonnent les pourtours des zones limitrophes. Les zones B3, B4 et B8, par exemple, jouxtaient les installations électrochimiques et électrométallurgiques des grandes firmes industrielles. Les fortes émanations qui s'en échappaient, il va sans dire, rendaient les habitations les plus touchées assez peu attrayantes. Mais de tous les éléments structurants de base, c'est peut-être la topographie qui a exercé, à cette échelle, l'influence la plus aisément perceptible. On chercherait en vain à Shawinigan de ces vastes perspectives qui permettent d'embrasser du seul regard l'essentiel du paysage. Point non plus de ces grandes avenues qui, droites et planes, laissent découvrir telle portion plus ou moins lointaine du territoire urbain. Ici, au contraire, tout est buttes et vallons, flancs de collines et ravins ${ }^{44}$. La grande section de la Pointeà-Bernard qui abrite le centre-ville est particulièrement accidentée, de même que ses abords. Outre le passage vers le secteur Saint-Onge, on ne peut y accéder que par deux voies permettant de franchir des obstacles naturels: un chemin plongeant dans une pente abrupte vers la municipalité proche de La Baie, et, depuis 1912, un pont franchissant le Saint-Maurice et menant à une municipalité distincte, aujourd'hui Shawinigan-Sud. Certaines zones résidentielles adjacentes sur la carte sont séparées par des flancs de collines et ne communiquent directement que peu ou pas du tout (A10, A11 et A12, ou B3 et B2 notamment). Que dire aussi de cette minuscule zone B9 sise au beau milieu de collines qui l'isolent presque complètement du reste de la ville...

Le processus de différenciation sociale de l'espace shawiniganais s'est donc appuyé fortement sur les caractéristiques topographiques du site. Qui plus est, ce n'est pas selon une logique neutre du point de vue social qu'il a adopté les formes physiques du paysage shawiniganais.

44. Le plan directeur préparé au tournant du siècle pour les dirigeants de SWP utilisait ces particularités du relief local: certains secteurs accidentés en particulier étaient prévus pour les groupes les plus aisés, et le caractère sinueux des rues qui y étaient dessinées tranchait avec celui du damier planifié pour le restant de la ville. Il ne faut pas s'en étonner, dans la mesure où le mouvement d'urbanisme dominant à l'époque en Amérique du Nord, le city beautiful movement, mettait notamment l'accent, dans une perspective paysagiste, sur la nécessité d'éviter la monotonie qu'engendrent des espaces urbains indifférenciés. Il faut reconnaître toutefois que le plan témoignait aussi d'une certaine méconnaissance des caractéristiques géomorphologiques du site, certaines rues ayant été dessinées à des endroits où elles ne pouvaient passer. 
Suivant en cela une rationalité dont on trouve des exemples un peu partout dans le monde, la plupart des emplacements situés en hauteur se sont vus appropriés par les éléments les plus influents de la société locale. Parions qu'il y aurait beaucoup à découvrir si l'on s'attardait davantage à cette véritable symbolique de la ville qui s'exprime de la façon la plus évidente dans la localisation privilégiée du petit secteur de la rue $\mathrm{Maple}^{45}$ (zone A11), et des sites choisis pour ériger l'église Saint-Pierre ou le luxueux hôtel Cascade Inn construit par les soins de SWP au tout début du siècle.

D'autres facteurs ont grandement contribué à façonner le visage de cette ville industrielle. La gestion du territoire par les administrations municipales en est un des plus importants à notre avis. Les élus municipaux et, dans une certaine mesure, le gérant municipal à partir de 1921, ont participé à l'inscription de la différence au sein de l'espace résidentiel à Shawinigan. Ils le firent notamment au cours des premières décennies en fournissant des services publics d'inégale qualité. Aussi, il semble que la réglementation adoptée vint bien souvent confirmer les différences sociales exprimées dans l'espace, les renforcer, leur assurer une certaine permanence, pourrait-on dire ${ }^{46}$. Le règlement de construction de $1922^{47}$ par exemple déterminait, pour chacune des rues de la ville, quelles catégories de bâtiments pouvaient être construits. Les immeubles de qualité inférieure n'étaient pas permis, il va sans dire, sur les rues commerçantes du centre-ville et dans les zones les plus aisées (Maple, par exemple). La catégorie la plus modeste n'était d'ailleurs autorisée essentiellement que dans les zones défavorisées du secteur Saint-Onge ${ }^{48}$. Une analyse fine de la réglementation municipale en matière de construction d'immeubles permettrait de préciser spatialement et chronologiquement la portée de ce phénomène. La carte 4 , qui, pour rendre compte des matériaux utilisés

45. Le nom de cette rue a depuis été francisé pour «des Érables».

46. J.-P. Collin a constaté que la réglementation adoptée dans quatre municipalités de l'ensemble montréalais de 1875 à 1920 contribuait à renforcer la ségrégation spatiale au sein de l'agglomération. Voir J.-P. Collin, «La cité sur mesure: spécialisation sociale de l'espace et autonomie municipale dans la banlieue montréalaise, 1875-1920», Revue d'histoire urbaine, 13,1 (juin 1984): 19-34. Il est généralement admis que les règlements de zonage assurent le développement de zones relativement homogènes.

47. Procès-verbaux des délibérations du conseil municipal, 19 avril 1922, règlement 225 .

48. À certains égards, cette réglementation s'inscrivait dans le prolongement des exigences exprimées dès le début du siècle dans les contrats de vente aux particuliers des terrains du secteur de la Pointe-à-Bernard, secteur à l'origine sous le plein contrôle de la compagnie d'électricité. À ce sujet, voir F. Guérard et G. Trépanier, «Shawinigan: une ville née de l'industrie», Continuité, 30 (hiver 1986). 


\section{CARTE 4 \\ LES MATÉRIAUX DE CONSTRUCTION À SHAWINIGAN SELON LA ZONE RÉSIDENTIELLE (1925 ET 1947)}

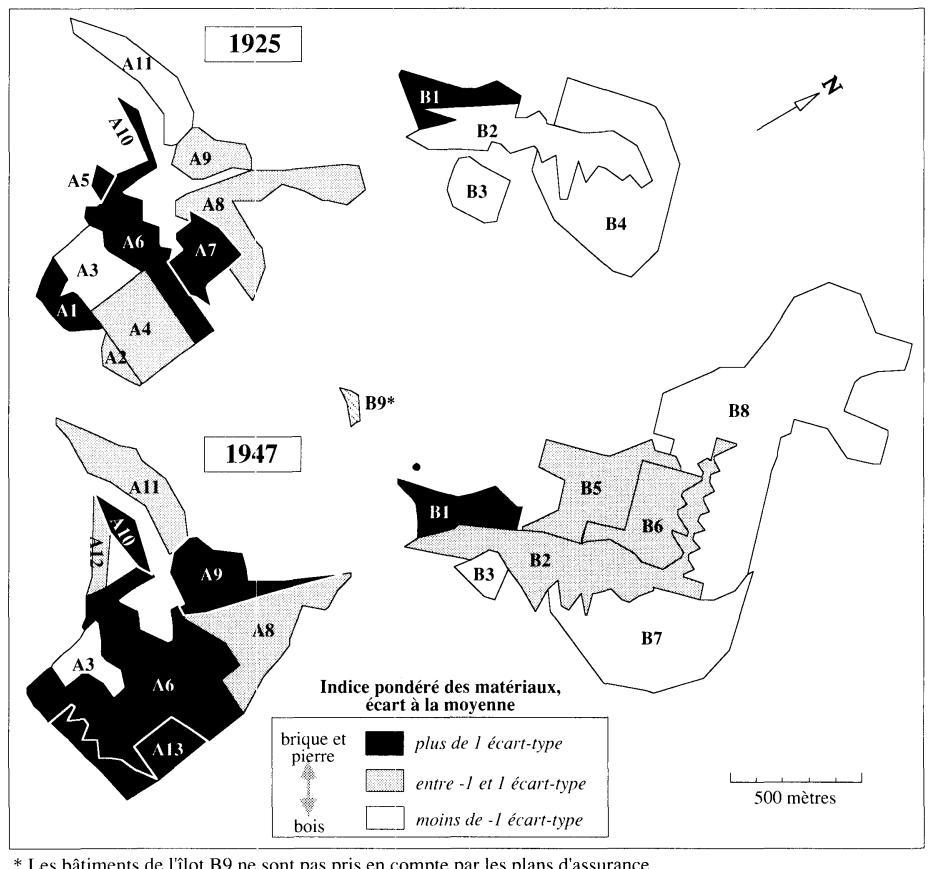

pour la construction des immeubles, recourt à un indice synthétique ${ }^{49}$, donne tout de même prise sur cette autre forme de disparité urbaine

49. Cet indice a été constitué à partir des informations sur les matériaux de charpente et de recouvrement inscrits aux plans d'assurances sous la forme d'un code de couleurs et de trames. Une cote numérique a été assignée à chacun des matériaux représentés dans la légende des plans d'assurance. Le bois, par exemple, s'est vu attribuer la cote «-1» alors que la brique et la pierre recevaient la note la plus élevée «1». D'autres matériaux, plus neutres pourrait-on dire, se sont vu accorder la note «0» (ciment, amiante, etc.). Ces matériaux ont souvent été combinés lors de la construction des bâtiments. La plupart des immeubles du centre-ville, par exemple, sont dessinés sur les plans d'assurance avec un périmètre rose (brique) et un centre jaune (bois). Aussi, a-t-il été nécessaire de définir un indice qui tienne compte de la diversité des matériaux utilisés de même que des phénomènes de dominance. De cette manière, pour reprendre le même exemple, les bâtiments faits de bois avec recouvrement de brique se sont vu attribuer la cote $« 0,75 »$. Les immeubles de pierre ont obtenu quant à eux la note «1» alors que les maisons entièrement en bois étaient classées «-1». Une cote d'ensemble a ensuite été calculée pour chacune des zones résidentielles en tenant compte de l'ensemble des bâtiments qui s'y trouvaient. Pour les besoins de la représentation cartographique, ces dernières valeurs ont été transformées à nouveau pour être représentées sous la forme d'écarts à la moyenne. 
qu'est la qualité inégale du logement ${ }^{50}$. On remarquera une fois encore cette nette opposition entre les deux grandes parties de la ville. Dès 1925, c'est dans le secteur de la Pointe-à-Bernard (A) que l'on retrouve presque toutes les zones où prédominent la brique et la pierre alors que le secteur Saint-Onge $(B)$ recèle 3 zones à dominance de bâtiments de bois - représentant $89,6 \%$ de sa surface - contre une seule zone de briques et de pierres ${ }^{51}$. Cette opposition s'affirme avec plus de netteté encore en 1947. Les zones teintées de noir couvrent alors environ $65 \%$ de la surface du grand secteur de la Pointe-àBernard. La seule zone à dominance de bois (A3) ne compte, elle, que pour un maigre $5,2 \%$. Du côté nord-est de la ville, on note une progression des matériaux les plus «nobles» dans les zones B2, B5 et B6 qui passent du blanc au gris. Le phénomène n'est pas assez important toutefois pour remettre en cause la primauté du bois comme matériau de recouvrement des bâtiments dans cette vaste partie où habitent les populations les moins bien nanties de Shawinigan.

$$
\text { * * * }
$$

Production de l'espace urbain. Le concept, large, englobant pourrait-on dire, renvoie d'abord à l'idée d'une structuration progressive d'un lieu, la ville. Il convie l'observateur à appréhender celleci non seulement dans ses cohérences internes mais aussi dans une perspective fondamentalement diachronique. La portée heuristique d'une telle approche est très grande. N'a-t-on pas affirmé avec beaucoup d'aplomb que l'espace urbain était une accumulation de temps ${ }^{52}$ ? Quoi qu'il en soit, c'est à telle enseigne que s'est logée la présente étude.

De toutes les fonctions urbaines, la résidentielle est sans doute celle par laquelle passe avec le plus de force le processus de la différenciation spatiale. Au terme de cette étude, il nous est apparu que l'espace résidentiel shawiniganais était socialement fortement diffé-

50. Le matériau de construction n'est toutefois qu'un indicateur de la qualité du logement qui gagne à être mis en rapport avec d'autres informations. Il existait, surtout au début du siècle, de magnifiques demeures construites en bois. On a érigé de telles habitations à Shawinigan, sur la rue Maple (zone A11), par exemple. Aussi, avons-nous jugé plus prudent ici de nous en tenir à des considérations générales. Des recherches plus précises prenant en compte le volume des logements, le style architectural, la présence d'espaces verts de même que l'ensemble des variables reliées au statut socio-professionnel de leurs habitants permettraient évidemment de raffiner considérablement l'analyse.

51. Il s'agit, on s'en souviendra, du secteur modèle créé par SWP et les autres grandes entreprises de la ville. Dans notre classification, il est apparenté aux secteurs A11 et A8.

52. Therry Paquot, «La grande métamorphose urbaine», Le Monde diplomatique, 458 (mai 1992). 
rencié. Mais encore a-t-il fallu, pour apprécier l'ampleur du phénomène et en saisir les formes précises, procéder à un découpage du territoire qui respecte les continuités au sein de l'espace résidentiel. On l'a vu, les cohérences du tissu urbain ne se laissent découvrir qu'à l'échelle appropriée. Qu'elle soit trop grande, ou trop petite, et l'observateur n'obtiendra qu'une image diffuse, difficile à interpréter $^{53}$. À notre avis, les méthodes mises en œuvre dans cette recherche pour délimiter des zones homogènes et pour en faire la classification pourraient ètre employées avec beaucoup de profit pour l'étude d'autres villes. Elles offriraient peut-être même quelques moyens pour des analyses comparatives mettant au jour les affinités entre les groupes sociaux ${ }^{54}$.

Plusieurs voies de recherches extrêmement intéressantes restent à explorer pour l'étude du cas de Shawinigan ${ }^{55}$. Nous terminerons cet article en présentant brièvement quelques avenues qui nous apparaissent particulièrement prometteuses. D'abord, la partition de l'espace résidentiel et la typologie des zones que nous proposons ici devraient permettre d'aborder dans le détail des questions importantes qui n'ont été qu'effleurées ici. Nous pensons notamment à l'accès inégal aux divers services publics et privés. La localisation des espaces verts à Shawinigan mériterait par exemple d'être confrontée avec la répartition des groupes ethniques et sociaux dans la ville. L'étude de la fonction commerciale à Shawinigan ne faisait pas partie à proprement parler de nos objectifs. Nous croyons cependant qu'on gagnerait beaucoup à enrichir la typologie des zones résidentielles en tenant compte de la qualité et de la diversité des services commerciaux.

Par ailleurs, faute de documents adéquats, un certain nombre de variables nous échappent. Nous pensons ici notamment aux revenus des ménages, au prix des loyers et à la localisation du lieu de travail. Peut-être sera-t-il possible de combler un jour ces lacunes. Il existe

53. Un peu, si l'on veut, comme le microscope du biologiste ou la lunette de l'astronome qui nécessitent une mise au point préalable. L'importance des phénomènes d'échelle est par ailleurs bien connue des géographes. Elle l'est par contre généralement beaucoup moins des historiens.

54. En plus de la classification hiérarchique, on pourrait mettre aussi à contribution l'analyse factorielle des correspondances. Rappelons que celle-ci permet de placer dans un plan imaginaire les modalités de diverses variables non numériques et non ordonnées en fonction de leur degré d'affinité.

55. C'est ce à quoi s'emploient les autres membres de l'équipe du projet dans le cadre duquel s'inscrit le présent article. Mentionnons, par exemple, les mémoires en cours de MarieJosée Dorion sur les ingénieurs et les techniciens shawiniganais et de Denise Deshaies-Fréchette sur la mobilité géographique dans le quartier Saint-Onge. 
enfin une autre approche pleine de promesses qui s'attacherait à découvrir l'inscription de la différence dans l'espace urbain par le biais cette fois du sentiment d'appartenance à un territoire de base (une section de rue ou un quartier, etc.) ${ }^{56}$. Nul doute que des enquêtes sur le terrain livreraient des résultats très stimulants. Certes, une connaissance approfondie de ces micro-espaces pour l'ensemble de la ville apparaît, dans l'état actuel des recherches, hors de portée. Nous croyons cependant que des études s'attachant à cerner les phénomènes de sociabilité - organisée ou spontanée - permettraient, à tout le moins, de mettre à l'essai le découpage en zones et la typologie proposés dans cet article.

56. Ce serait poser tout le problème de la territorialité. À ce sujet, voir Claude Raffestin, Pour une géographie du pouvoir (Paris, Litec, 1980), et Marcel Roncayolo, op. cit. 
APPENDICE 1

LES CATÉGORIES SOCIO-PROFESSIONNELLES À L'INTÉRIEUR DES ZONES RÉSIDENTIELLES: LES ÉCARTS PAR RAPPORT À UNE DISTRIBUTION HOMOGÈNE HISTOGRAMMES PAR ZONE

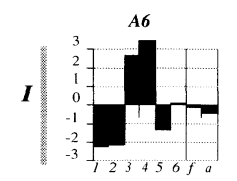

1925

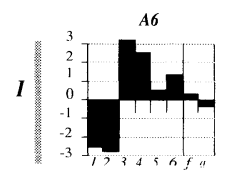

1947
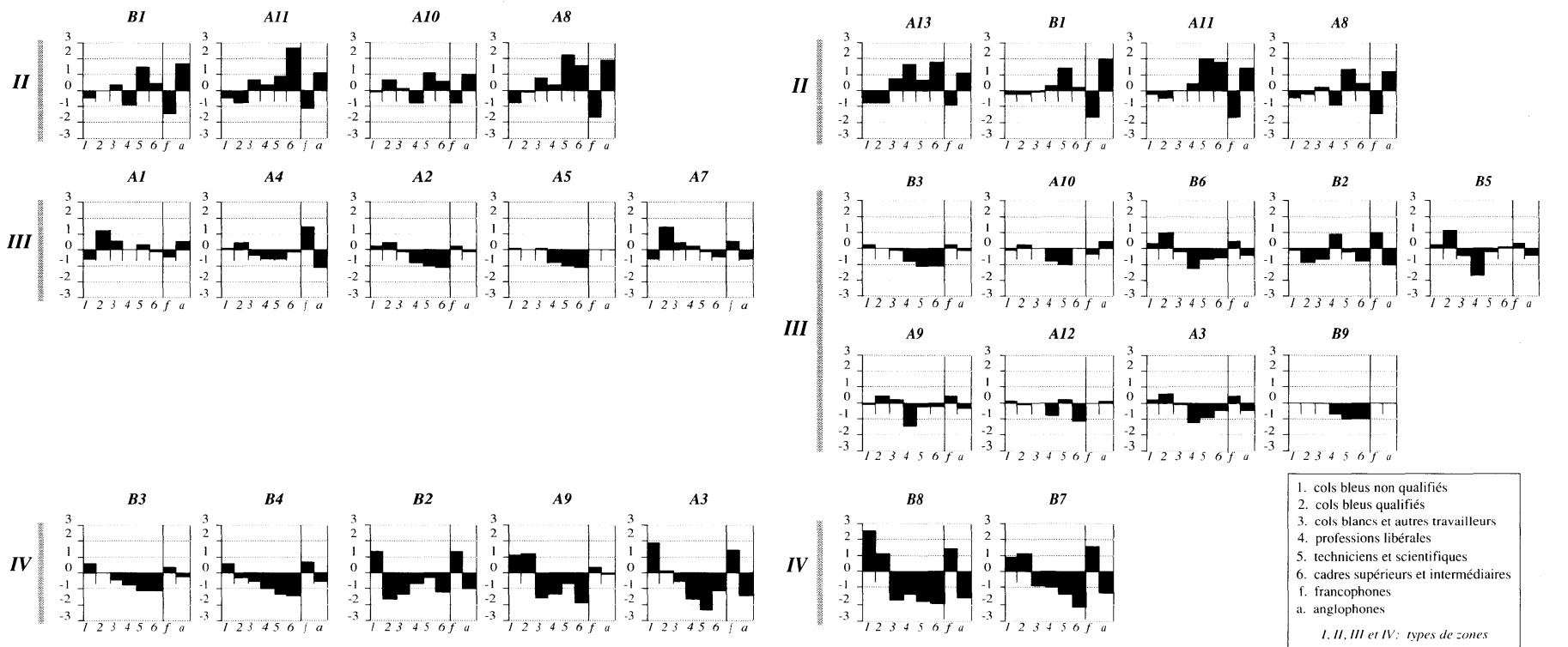
APPENDICE 2

SHAWINIGAN, 1925 ET 1947

LES CATÉGORIES SOCIO-PROFESSIONNELLES À L'INTÉRIEUR DES ZONES RÉSIDENTIELLES: LES ÉCARTS PAR RAPPORT À UNE DISTRIBUTION HOMOGÈNE HISTOGRAMMES PAR CATÉGORIE SOCIO-PROFESSIONNELLE

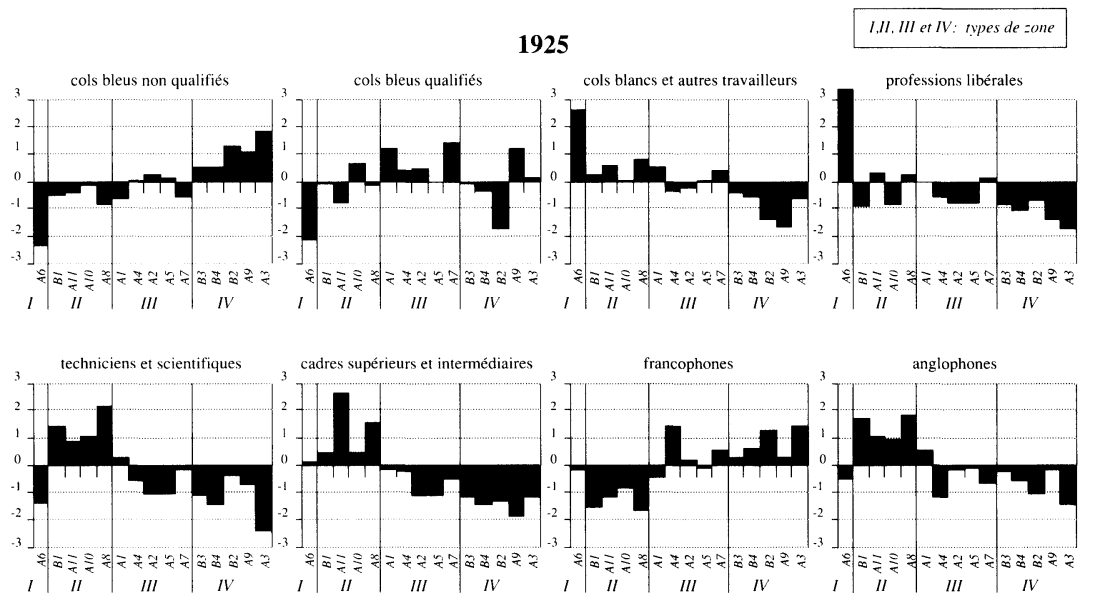

1947
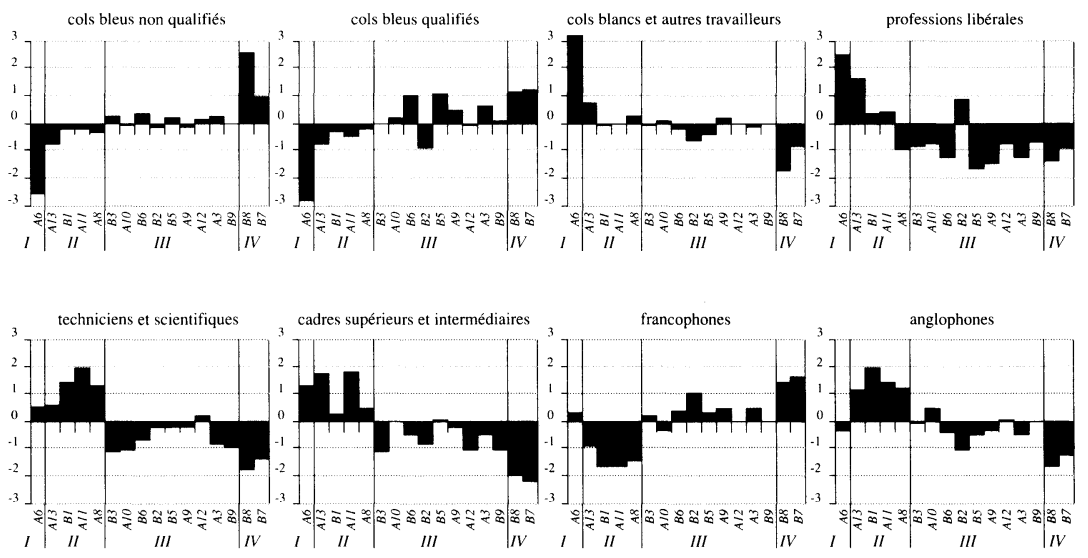